\title{
A Nano- and Micro- Integrated Protein Chip Based on Quantum Dot Probes and a Microfluidic Network
}

\author{
Juan Yan ${ }^{1}$, Mei Hu² ${ }^{2}$ Di Li ${ }^{1}$, Yao He ${ }^{1,2}$, Rui Zhao ${ }^{3}$, Xingyu Jiang ${ }^{3}$, Shiping Song ${ }^{1}(\varangle)$, Lianhui Wang ${ }^{2}(\varangle)$, \\ and Chunhai $\operatorname{Fan}^{1}(\bowtie)$ \\ ${ }^{1}$ Shanghai Institute of Applied Physics, Chinese Academy of Sciences, Shanghai 201800, China \\ ${ }^{2}$ Laboratory of Advanced Materials (LAM), Fudan University, 220 Handan Road, Shanghai 200433, China \\ ${ }^{3}$ National Center for NanoScience and Technology, No. 11, Beiyitiao, Zhongguancun, Beijing 100080, China \\ Received: 19 August 2008 / Revised: 3 November 2008 / Accepted: 3 November 2008 \\ (C) Tsinghua Press and Springer-Verlag 2008. This article is published with open access at Springerlink.com
}

\begin{abstract}
A novel nano- and micro-integrated protein chip (NMIPC) that can detect proteins with ultrahigh sensitivity has been fabricated. A microfluidic network $(\mu \mathrm{FN})$ was used to construct the protein chips, which allowed facile patterning of proteins and subsequent biomolecular recognition. Aqueous phase-synthesized, water-soluble fluorescent CdTe/CdS core-shell quantum dots (aqQDs), having high quantum yield and high photostability, were used as the signaling probe. Importantly, it was found that aqQDs were compatible with microfluidic format assays, which afforded highly sensitive protein chips for cancer biomarker assays.
\end{abstract}

\section{KEYWORDS}

Quantum dots, microfluidics, carcinoma embryonic antigen (CEA), microarray, polydimethylsiloxane (PDMS)

\section{Introduction}

Protein microarrays [1-3] have shown great promise in both basic and applied biological research [4-7]. This miniaturized multi-target analysis technology is particularly useful in molecular diagnostics, e.g., early-stage detection of tumor biomarkers in blood sera, cell lines and on the membranes of blood cells [7]. However, protein microarray technology is still faced with several challenge [8], such as relatively low sensitivity.

In this regard, semiconductor quantum dots (QDs) have shown unprecedented advantages, such as high quantum yield and photostability, and size-dependent tunable photoluminescence with narrow emission bandwidth and broad excitation spectra [9-12]. During the past two decades, considerable advances have been made in the areas of synthesis and biological applications of high quality QDs [13-18]. For example, Baba and coworkers have demonstrated that QDs bring about significantly improved sensitivity in western blotbased protein detection [19], which potentially means that the information from low abundance proteins could be elucidated. Nevertheless, in order to meet the requirements of biological applications, QDs synthesized in an organic phase have to be transferred to aqueous solution, which is rather complicated and often associated with significant losses of both photoluminescence (PL) quantum

Address correspondence to Shiping Song, spsong@sinap.ac.cn; Lianhui Wang, wlhui@fudan.edu.cn; Chunhai Fan, fchh@sinap.ac.cn 
yield (QY) and stability [20-22]. In order to avoid these problems, we recently developed a highly efficient program process of microwave irradiation (PPMI) strategy that allows the synthesis of highly fluorescent water-dispersed QDs directly from aqueous solution (aqQDs) [23, 24].

Another major obstacle with microarrays lies in the complex fabrication process that requires expensive automated spotting robots as well as strict reaction conditions such as humidity and temperature control [2, 25-27]. Microfluidic technology has proven to be a promising solution that offers convenient operation for both protein immobilization and subsequent binding assays [28-33]. In this contribution, we describe a novel nano- and microintegrated protein chip (NMIPC) that integrates the advantages of both microfluidic networks $(\mu \mathrm{FN})$ and aqQDs, and successfully demonstrates the combined advantages of using both aqQDs probes and a $\mu \mathrm{FN}$ by using carcinoma embryonic antigen (CEA) as a model assay target. This novel NMIPC system offers ultrahigh sensitivity for protein assays as well as convenience in both chip fabrication and detection, and is a potentially promising tool for portable pointof-care (POC) medical diagnostics.

\section{Results and discussion}

Capture antibodies (monoclonal antibodies, $\mathrm{mAb}$ ) were first passed through the microfluidic channels in the first polydimethylsiloxane (PDMS) layer, which resulted in their immobilization on the aldehyde-activated slide (see the scheme in Fig. 1). Then the remaining area of the slide was blocked with bovine serum albumin (BSA) to prevent nonspecific adsorption in subsequent steps and thus reduce the background signal. After this step, the first PDMS layer was peeled off and a second PDMS layer was attached to the slide, resulting in a crossedchannel configuration (see details in Fig. S-1 in the Electronic Supplementary Material, ESM). Target proteins were then passed through these channels, crossing the original capture mAb-bound channels, forming the $\mu \mathrm{FN}$. It should be noted that the target proteins were captured in the cross-section of the $\mu \mathrm{FN}$. After binding, biotinylated detection antibodies (monoclonal antibodies, $\mathrm{mAb}$ ) were passed through the same channels, followed by the signaling probe, avidin-coated $\mathrm{CdTe} / \mathrm{CdS}$ core-shell aqQDs, that illuminates the protein binding assays (see Fig. S-1 in the ESM for details of the device construction).

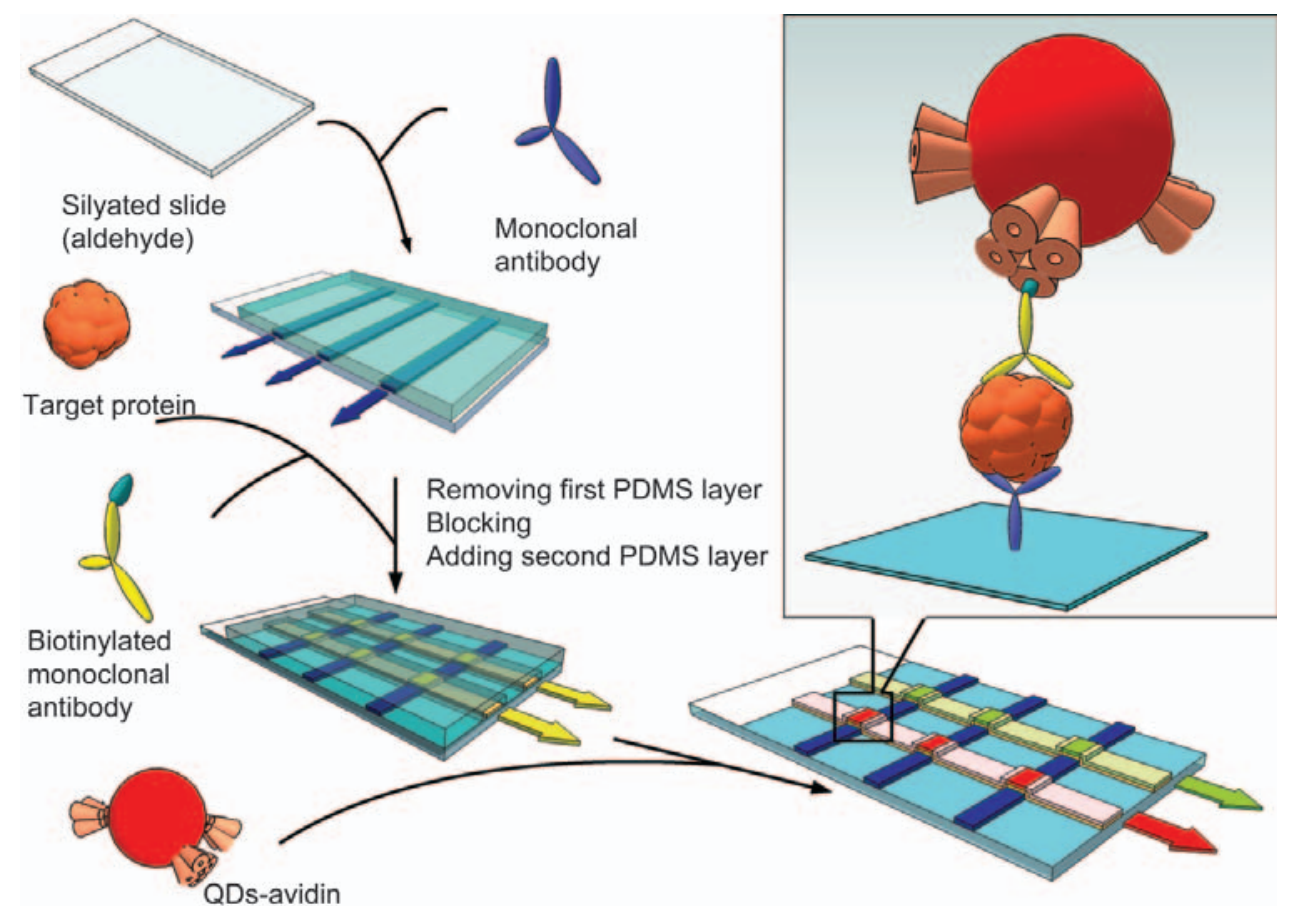

Figure 1 Schematic illustration of protein chips based on aqQDs probes and microfluidic network 


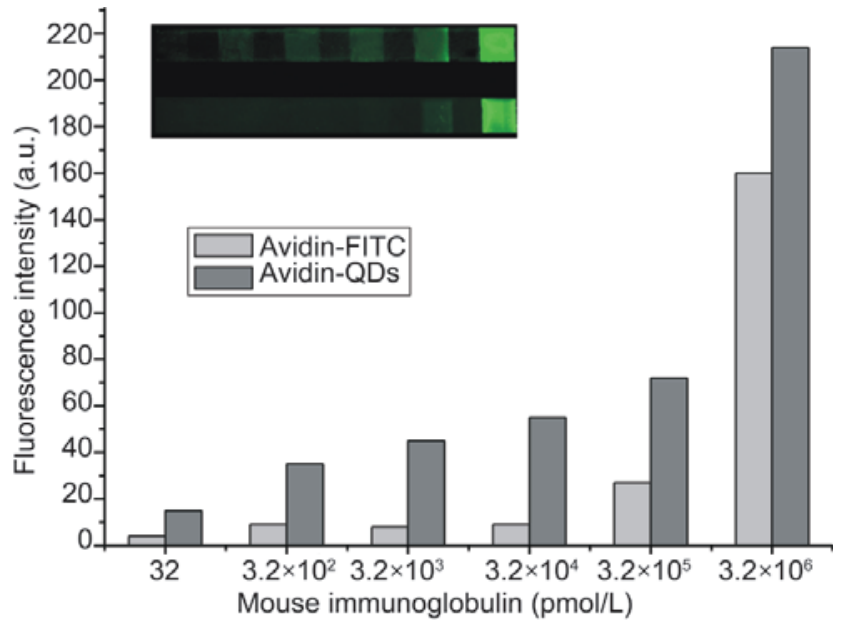

Figure 2 Comparison of the brightness of avidin-QDs and avidin-FITC as signal probes. Inset: the fluorescence images of the microfluidic network arrays using the two fluorescent probes with the target protein concentration range from $3.2 \times 10^{-6} \mathrm{~mol} / \mathrm{L}$ to $3.2 \times$ $10^{-11} \mathrm{~mol} / \mathrm{L}$, respectively (up: avidin-QDs, bottom: avidin-FITC)

We first tested the sensitivity of the NMIPC by using different concentrations of capture antibodies (mouse IgG), with either avidin-QDs or avidinfluorescein isothiocyanate (FITC) as the signaling probe. Avidin-QDs possess approximately the same fluorescence properties as aqQDs, and remain well dispersed in solution (Figs. S-2 and S-3 in the ESM). In addition, a biotinylated polyclonal antibody (goat anti-mouse $\operatorname{IgG}$ ) was employed as a ligand which can bind to both mouse IgG and avidin. As shown in Fig. 2 , the green-colored avidin-QDs probe $\left(\lambda_{\mathrm{em}}=554\right.$ $\mathrm{nm}$ ) exhibited a concentration-dependent change in fluorescence intensity in the range $32 \mathrm{pmol} / \mathrm{L}$ $-3.2 \mu \mathrm{mol} / \mathrm{L}$. In contrast, the organic dye avidin-
FITC [34], afforded significantly lower fluorescence intensities. In the latter case, the signal could not be distinguished from the background when the target concentration was below $320 \mathrm{nmol} / \mathrm{L}$ (four orders of magnitude higher than the corresponding value for aqQDs). This comparison clearly demonstrates that the aqQDs probe is fully compatible with the microfluidic format and affords ultrahigh sensitivity.

It is known that different colored QDs can be excited with a single wavelength due to their broad excitation bands. As shown in Fig. 3, protein chips labeled with aqQDs of two different colors could be effectively excited with a single laser source, implying that QDs-based protein chips allow convenient multiplex protein detection. In addition, QDs also showed high stability toward photobleaching. Figure 3 compares the photostability of FITC (A) and two kinds of aqQDs, B and C (with $\lambda_{\mathrm{em}}$ of $554 \mathrm{~nm}$ and $579 \mathrm{~nm}$, respectively) on protein chips. Clearly, fluorescent signals from FITC (A1) largely diminished after being irradiated with blue light ( $\lambda=450-490 \mathrm{~nm}$ ) for $30 \mathrm{~min}$ (A2). In contrast, signals from both aqQDs decreased by only a small degree (the discrepancy of the background contrast results from the different excitation wavelengths for FITC and QDs, and possibly also from QD diffusion on the surface of the microchannels). In fact, spots for the two QDs probes were still visible even after irradiation for $4 \mathrm{~h}$ (data not shown). This high photostability ensures the signal reproducibility of our protein chips.

In order to demonstrate the application of the NMIPC system in molecular diagnostics, we
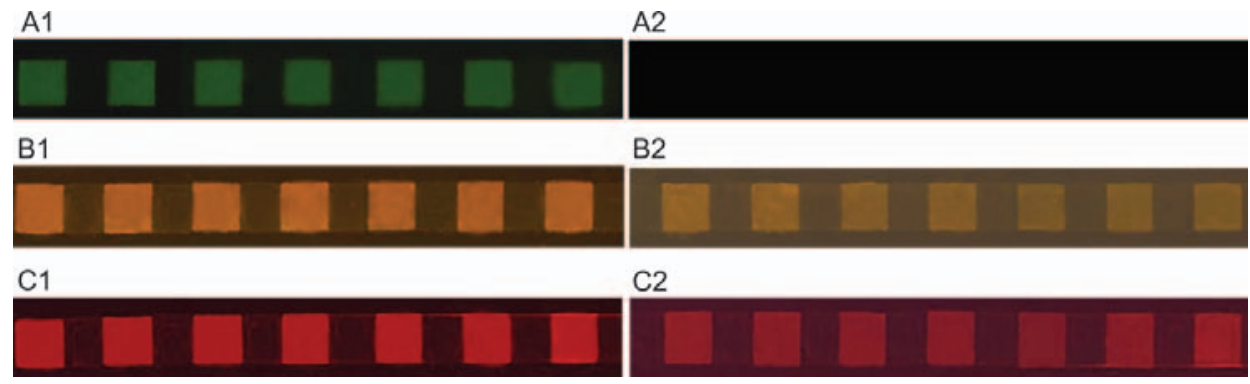

Figure 3 Photostability of avidin-QDs and avidin-FITC. A1: fluorescence images of microfluidic arrays using avidin-FITC probes. B1 and C1: fluorescence images of microfluidic arrays using two avidin-QDs with emission band at $554 \mathrm{~nm}$ and $579 \mathrm{~nm}$, respectively. A2, B2 and C2: corresponded fluorescence image after 30 min photobleaching. In all experiments, monoclonal antibodies were directly immobilized on the protein chip, biotinylated secondary antibodies, avidin-QDs or avidin-FITC probes were associated on the modified chip by flowing through the channels 
designed a $\mu \mathrm{FN}$ chip for a tumor marker, CEA, with a sandwich-based configuration as shown in Fig. 1. Avidin-coated aqQDs $\left(\lambda_{\mathrm{em}}=579 \mathrm{~nm}\right)$ were used as a signaling probe for this assay. Figure 4 displays a typical dose-response result for CEA over a wide concentration range covering six orders of magnitude, from $500 \mathrm{nmol} / \mathrm{L}$ to $500 \mathrm{fmol} / \mathrm{L}$. The limit of detection (LOD) was estimated to be $500 \mathrm{fmol} / \mathrm{L}$ (see Fig. S-4 in the ESM). In contrast, protein chips using avidin-FITC as the signaling probe showed a detectable signal only when the target concentration reached $500 \mathrm{nmol} / \mathrm{L}$. Moreover, the fluorescence signal for $500 \mathrm{fmol} / \mathrm{L} \mathrm{CEA} \mathrm{was}$ significantly higher than that of alpha-fetoprotein (AFP, negative control) with a concentration of $15 \mathrm{nmol} / \mathrm{L}$, indicating that our NMIPC system possesses high detection specificity (see Fig. S-4 in the ESM). It is important to note that since avidinQDs remain highly fluorescent in serum (Figs. S-5 and S-6 in the ESM), this QD-based protein chip should find applications in assays in serum or even whole blood.

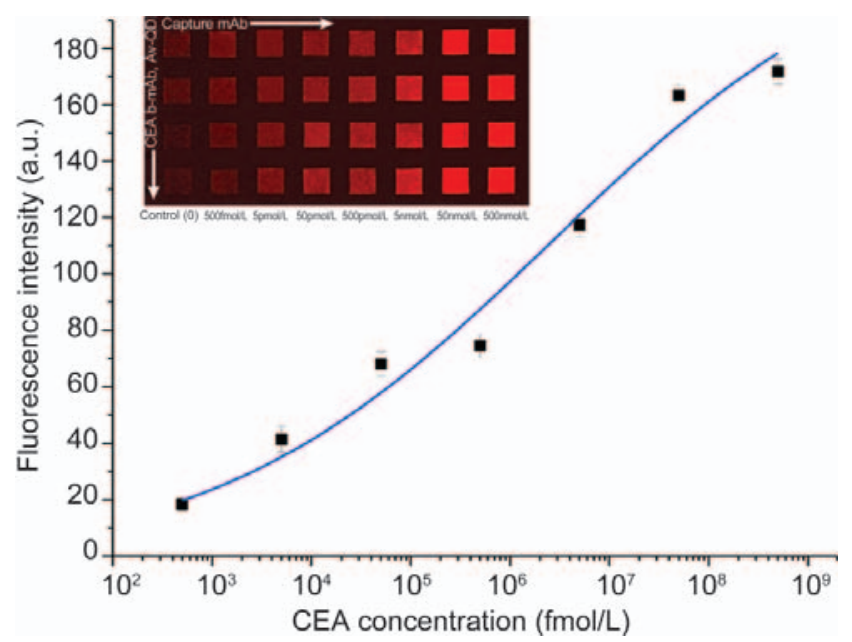

Figure 4 The dose-response calibration curve for CEA based on the aqQDs probe $\left(\lambda_{\mathrm{em}}=579 \mathrm{~nm}\right)$ and microfluidic network chip. The standard curve was fitted by a logistic model (Equation: $y=225.18 \pm 29.21+(-3.43 \pm 12.45-225.18 \pm 29.21) /(1+$ $(x / 2529665.20 \pm 2043790.60) 0.26 \pm 0.06), R 2=0.99)$. Backgroundsubtracted fluorescence intensity was used as the index. Insert (upper left): fluorescent image of parallel detection of CEA samples (Concentrations: 0, $500 \mathrm{fmol} / \mathrm{L}, 5 \mathrm{pmol} / \mathrm{L}, 50 \mathrm{pmol} / \mathrm{L}, 500 \mathrm{pmol} / \mathrm{L}, 5$ $\mathrm{nmol} / \mathrm{L}, 50 \mathrm{nmol} / \mathrm{L}, 500 \mathrm{nmol} / \mathrm{L}$ ) in four parallel microfluidic channels. Arrowheads indicate the flow directions

\section{Methods}

\subsection{Materials}

Silyated slides (aldehyde activated) were obtained from CEL Associates (Pearland, USA). Sylgard184 was purchased from Dow Corning Co. Ltd. (Shanghai, China). All antigens (target proteins) were purchased from Industries International (Concord, USA). Monoclonal antibodies and biotinylated monoclonal antibodies were purchased from USBIO. Biotinylated secondary antibody (anti-mouse polyclonal antibody), avidin, avidin-FITC, BSA, tellurium powder (99.9\%), and $\mathrm{CdCl}_{2}(99.9 \%)$ were purchased from Sigma-Aldrich (St. Louis, MO, USA). Tween 20 and 3-mercaptopropionic acid (MPA) (98\%) were purchased from Fluka. Silicon patterns were fabricated by Shanghai Institute of Microsystem and Information Technology (SIMIT), Chinese Academy of Sciences. The syringe pump was obtained from Zhejiang University.

\subsection{Fabrication of microfluidic channels}

Polydimethylsiloxane layers containing 22 microchannels were prepared by contact photolithography using silicon patterns as molds. The microchannels had a width of $150 \mu \mathrm{m}$ and a height of $30 \mu \mathrm{m}$ with a gap of $150 \mu \mathrm{m}$ between the channels.

\subsection{Synthesis of water-dispersed CdTe/CdS core- shell aqQDs}

Nearly monodispersed CdTe/CdS core-shell aqQDs were obtained via microwave irradiation as described in our previous work [24]. Briefly, a CdTe precursor solution was prepared by adding freshly prepared $\mathrm{NaHTe}$ solution to $\mathrm{N}_{2}$-saturated $\mathrm{CdCl}_{2}$ solution at pH 8.4 in the presence of 3-mercaptopropionic acid (MPA) as the stabilizer. The precursor concentrations were $[\mathrm{Cd}]=1.25 \mathrm{mmol} / \mathrm{L}$, $[\mathrm{MPA}]=3.0 \mathrm{mmol} / \mathrm{L}$, and $[\mathrm{Te}]=0.625 \mathrm{mmol} / \mathrm{L}$. The CdTe precursor solution (50 $\mathrm{mL}$ ) was injected into glass bottle. CdTe nanocrystals (NCs) with a maximum emission wavelength of around $520 \mathrm{~nm}$ were prepared under microwave irradiation for $1 \mathrm{~min}$ at $100{ }^{\circ} \mathrm{C}$. The CdTe NCs sample was removed when the temperature cooled to lower than $50{ }^{\circ} \mathrm{C}$ at room temperature. The as-

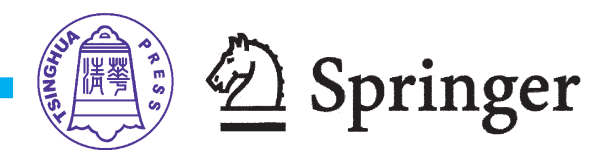


prepared CdTe solution was reduced to one quarter of its original volume, and CdTe then precipitated with 2-propanol and separated by centrifugation. The colloidal precipitate was redissolved in $3 \mathrm{~mL}$ of ultrapure water, to give CdTe core NCs. The CdTe/ CdS precursor solution was prepared by adding the as-prepared CdTe core NCs to a $\mathrm{N}_{2}$-saturated solution with the concentrations of $1.25 \mathrm{mmol} / \mathrm{L}$ $\mathrm{CdCl}_{2}, 1.0 \mathrm{mmol} / \mathrm{L} \mathrm{Na}_{2} \mathrm{~S}$, and $6.0 \mathrm{mmol} / \mathrm{L} \mathrm{MPA}$ at $\mathrm{pH}$ 8.4. The CdTe/CdS precursor solution $(4 \mathrm{~mL})$ was injected into the exclusive vitreous vessel. A series of high quality CdTe/CdS NCs with different sizes were obtained by regulating the time of microwave irradiation. After microwave irradiation, the CdTe/ CdS NCs sample was removed when the temperature cooled to lower than $50{ }^{\circ} \mathrm{C}$ at room temperature. No post-preparative treatment was performed on any of the samples prior to optical characterization. Samples were precipitated by 2-propanol and dried in a vacuum oven for XRD and XPS characterization. TEM, HRTEM, and EDAX samples were prepared by dropping the aqueous $\mathrm{CdTe} / \mathrm{CdS}$ solution onto carbon-coated copper grids with the excess solvent being evaporated. The PL QY at room temperature was estimated using rhodamine 6G (R6G) (QY=95\%), rhodamine $640(\mathrm{R} 640)$ (QY=100\%), or Oxazine 4 (LD690) (QY=63\%) in ethanol, according to the different maximum emission wavelengths of the CdTe/CdS core-shell NCs.

\subsection{Preparation and validation of avidin linked aqQDs probes}

The conjugation of MPA-capped aqQDs with avidin was performed as previously reported [10, 35, 36]. Avidin, a positively charged protein, is able to interact with the negatively charged MPA-capped QDs. An optimal protocol is as follows. QDs (1.2 $\mathrm{nmol})$ and avidin $(1.2 \mathrm{nmol})$ were mixed and the final volume of mixture was brought to $600 \mu \mathrm{L}$ using PBS buffer ( $\mathrm{pH} 7.4,150 \mathrm{mmol} / \mathrm{L} \mathrm{Na}_{2} \mathrm{HPO}_{4} \cdot 12 \mathrm{H}_{2} \mathrm{O}, 150$ $\mathrm{mmol} / \mathrm{L} \mathrm{KH}_{2} \mathrm{PO}_{4}, 136 \mathrm{mmol} / \mathrm{L} \mathrm{NaCl}$, and $2 \mathrm{mmol} /$ $\mathrm{L} \mathrm{KCl}$ ). The mixture was incubated in the dark at room temperature for $30 \mathrm{~min}$ and then ultrafiltered using 30K Nanosep centrifugal devices at 5000 rpm for $20 \mathrm{~min}$. The filtrate containing free nonconjugated QDs was then removed. The upper phase containing QDs-avidin conjugates was concentrated to about $10 \mu \mathrm{mol} / \mathrm{L}$. These conjugates showed a bright photoluminescence and were stored at $4{ }^{\circ} \mathrm{C}$ in the dark for subsequent use. For the preparation of multicolored avidin linked QDs probes, two kinds of QDs with different sizes were used: one with the maximum emission wavelength of $554 \mathrm{~nm}$ (orange under UV irradiation) and the other with the maximum emission wavelength of $579 \mathrm{~nm}$ (red under UV irradiation).

Mouse immunoglobulin was dissolved in PBS (150 mmol/L, pH 7.0, 40\% glycerol) at a series of concentrations $(32 \mathrm{pmol} / \mathrm{L}-3.2 \mu \mathrm{mol} / \mathrm{L})$. The solutions were immobilized on the chip through microchannels of the first PDMS layer and incubated at $37{ }^{\circ} \mathrm{C}$ for $1 \mathrm{~h}$. The chip was then washed with PBS containing $0.25 \%$ Tween 20 and blocked with PBS containing 5\% BSA. Then biotinylated antimouse polyclonal antibody with concentration 0.37 $\mathrm{mg} / \mathrm{mL}(10 \mu \mathrm{L}$ of $3.7 \mathrm{mg} / \mathrm{mL}$ antibody mixed with $25 \mu \mathrm{L} 20 \%$ BSA and $65 \mu \mathrm{L}$ PBS of $150 \mathrm{mmol} / \mathrm{L}$ at $\mathrm{pH}$ 7.0) was passed into the slide through the second PDMS layer. The antibody recognized and bound to the mouse immunoglobulin at cross sections of the microchannels in the first and second PDMS layers. After incubation at $37^{\circ} \mathrm{C}$ for $1 \mathrm{~h}$, the chip was carefully rinsed to remove unbound biotinylated secondary antibody. QDs-conjugated avidin (or FITCconjugated avidin) was used as a signaling probe. After incubation for $15 \mathrm{~min}$, fluorescent signals were fixed with GSH and the resulting array was imaged using fluorescence microscopy. An additional photobleaching test was performed to compare the photostability of the QDs probe with that of organic dyes.

\subsection{Detection of tumor markers using protein chips}

The first monoclonal antibodies (C1300-02, 0.5 $\mathrm{mg} / \mathrm{mL}$, dissolved in PBS of concentration 150 $\mathrm{mmol} / \mathrm{L}$, pH 7.4, 40\% glycerol) were deposited on the aldehyde-activated chip base through the first PDMS layer. After incubation at $37{ }^{\circ} \mathrm{C}$ for $1 \mathrm{~h}$, the microchannels were rinsed and then the first PDMS layer was peeled off. The slide was rinsed at least three times with the washing buffer $(150 \mathrm{mmol} / \mathrm{L}$ PBS, $0.25 \%$ Tween 20) and blocked with the blocking 
buffer (150 mmol/L PBS, pH 7.4, 5\% BSA) for 30 min. A second PDMS layer was then attached to the surface of the slide, with microchannels crossing the first pattern (Fig. S-1 in the ESM). Then a range of concentrations $(500 \mathrm{fmol} / \mathrm{L}-500 \mathrm{nmol} / \mathrm{L}$, dissolved in PBS (150 mmol/L, pH 7.4, 2\% BSA)) of CEA solutions was passed through the second PDMS layer. After incubation at $37^{\circ} \mathrm{C}$ for $1 \mathrm{~h}$ and repeated rising steps, biotinylated monoclonal antibody (C1300-07A, $0.2 \mathrm{mg} / \mathrm{mL}$ ) was introduced to the cross areas where they bound to the target protein. After a rinsing step that removed unbound antibodies, avidin-coated aqQDs were used to probe the binding process. After $15 \mathrm{~min}$, fluorescence signals were further fixed by a solution of reduced L-glutathione (GSH). Finally, the second PDMS layer was removed and the chips were imaged with a fluorescence microscope (AxioSkop, ZEISS) equipped with optical filters and a chargecoupled camera (AxioCam MRC5, ZEISS). Signals were excited at $365 \mathrm{~nm} \pm 12 \mathrm{~nm}$ and $450-490 \mathrm{~nm}$ (power: $100 \mathrm{~W}$; gain: 1; exposure time: $1028 \mathrm{~ms}$ ). Fluorescence intensity was calculated from the image using software written in-house.

\section{Conclusions}

In summary, we have successfully demonstrated a novel NMIPC system for protein detection, which has several advantages. Firstly, the use of aqQDs as the signaling probe offers high quantum yield and high photostability, which leads to highly sensitive and robust protein chips. Secondly, the broad excitation band of aqQDs offers the potential for multiplex detection within a single chip. Thirdly, the use of a $\mu \mathrm{FN}$ avoids expensive automated spotting robots and provides an ideal environment for protein binding assays, including the ease of sample delivery and protein binding. We thus expect this NMIPC system will be a promising tool for molecular diagnostics and proteomics.

\section{Acknowledgements}

This work was supported by the National Natural Science Foundation (20873175 and 20725516), the Ministry of Science and Technology (2006CB933000,
2007CB936000, and 2007AA06A406), and the Shanghai Municipal Commission for Science and Technology (0852nm00400, 0752nm021).

Electronic Supplementary Material: Six figures describing the construction of the microfluidic network, limits of detection for CEA assays, fluorescence and light scattering of QDs before and after conjugation with avidin, and fluorescence spectra and white light and fluorescence imaging of QDs-avidin in PSB and serum are available in the online version of this article at http://dx.doi. org/10.1007/s12274-008-8052-1 and are accessible free of charge.

\section{References}

[1] Ekins, R.; Chu, F. W. Microarrays: Their origins and applications. Trends Biotechnol. 1999, 17, 217-218.

[2] MacBeath, G.; Schreiber, S. L. Printing proteins as microarrays for high-throughput function determination. Science 2000, 289, 1760-1763.

[3] Lueking, A.; Horn, M.; Eickhoff, H.; Bussow, K.; Lehrach, H.; Walter, G. Protein microarrays for gene expression and antibody screening. Anal. Biochem. 1999, 270, 103-111.

[4] Hultschig, C.; Kreutzberger, J.; Seitz, H.; Konthur, Z.; Bussow, K.; Lehrach, H. Recent advances of protein microarrays. Curr. Opin. Chem. Biol. 2006, 10, 4-10.

[5] Sauer, S.; Lange, B. M.; Gobom, J.; Nyarsik, L.; Seitz, H.; Lehrach, $\mathrm{H}$. Miniaturization in functional genomics and proteomics. Nat. Rev. Genet. 2005, 6, 465-476.

[6] Templin, M. F.; Stoll, D.; Schwenk, J. M.; Potz, O.; Kramer, S.; Joos, T. O. Protein microarrays: Promising tools for proteomic research. Proteomics 2003, 3, 2155-2166.

[7] Haab, B. B. Methods and applications of antibody microarrays in cancer research. Proteomics 2003, 3, 2116-2122.

[8] Eisenstein, M. Protein arrays - Growing pains. Nature 2006, 444, 959-962.

[9] Bruchez, M., Jr.; Moronne, M.; Gin, P.; Weiss, S.; Alivisatos, A. P. Semiconductor nanocrystals as fluorescent biological labels. Science 1998, 281, 2013-2016.

[10] Chan, W. C.; Nie, S. Quantum dot bioconjugates for ultrasensitive nonisotopic detection. Science 1998, 281, 2016-2018. 
[11] Medintz, I. L.; Uyeda, H. T.; Goldman, E. R.; Mattoussi, H. Quantum dot bioconjugates for imaging, labelling and sensing. Nat. Mater. 2005, 4, 435-446.

[12] Michalet, X.; Pinaud, F. F.; Bentolila, L. A.; Tsay, J. M.; Doose, S.; Li, J. J.; Sundaresan, G.; Wu, A. M.; Gambhir, S. S.; Weiss, S. Quantum dots for live cells, in vivo imaging, and diagnostics. Science 2005, 307, 538-544.

[13] Peng, X. G.; Manna, L.; Yang, W. D.; Wickham, J.; Scher, E.; Kadavanich, A.; Alivisatos, A. P. Shape control of CdSe nanocrystals. Nature 2000, 404, 59-61.

[15] Alivisatos, A. P. Semiconductor clusters, nanocrystals, and quantum dots. Science 1996, 271, 933-937.

[16] Battaglia, D.; Peng, X. G. Formation of high quality InP and InAs nanocrystals in a noncoordinating solvent. Nano Lett. 2002, 2, 1027-1030.

[17] Tang, Z. Y.; Kotov, N. A.; Giersig, M. Spontaneous organization of single CdTe nanoparticles into luminescent nanowires. Science 2002, 297, 237-240

[18] Qu, L.; Peng, X. G. Control of photoluminescence properties of CdSe nanocrystals in growth. J. Am. Chem. Soc. 2002, 124, 2049-2055.

[19] Bakalova, R.; Zhelev, Z.; Ohba, H.; Baba, Y. Quantum dot-based western blot technology for ultrasensitive detection of tracer proteins. J. Am. Chem. Soc. 2005, 127, 9328-9329.

[20] Wuister, S. F.; Swart, I.; Driel, F. V.; Hickey, S. G.; Donega, C. M. Highly luminescent water-soluble CdTe quantum dots. Nano Lett. 2003, 3, 503-507.

[21] Wang, Y.; Tang, Z.; Correa-Duarte, M. A.; PastorizaSantos, I.; Giersig, M.; Kotov, N. A.; Liz-Marzan, L. M. Mechanism of strong luminescence photoactivation of citrate-stabilized water-soluble nanoparticles with CdSe cores. J. Phys. Chem. B 2004, 108, 15461-15469.

[22] Bao, H.; Gong, Y.; Li, Z.; Gao, M. Enhancement effect of illumination on the photoluminescence of water-soluble CdTe nanocrystals: Toward highly fluorescent $\mathrm{CdTe/CdS}$ core-shell structure. Chem. Mater. 2004, 16, 38533859.

[23] He, Y.; Lu, H.; Sai, L.; Su, Y.; Hu, M.; Fan, C.; Huang, W.; Wang, L. Enhancement effect of illumination on the photoluminescence of water-soluble CdTe nanocrystals: Toward highly fluorescent CdTe/CdS core-shell structure. Adv. Mater. 2008, 20, 3416-3421.

[24] He, Y.; Lu, H.; Sai, L.; Lai, W.; Fan, Q.; Wang, L.; Huang, W. Microwave-assisted growth and characterization of water-dispersed CdTe/CdS core-shell nanocrystals with high photoluminescence. J. Phys. Chem. B 2006, 110, 13370-13374.

[25] Balboni, I.; Chan, S. M.; Kattah, M.; Tenenbaum, J. D.; Butte, A. J.; Utz, P. J. Multiplexed protein array platforms for analysis of autoimmune diseases. Annu.Rev. Immunol. 2006, 24, 391-418.

[26] Chan, S. M.; Ermann, J.; Su, L.; Fathman, C. G.; Utz, P. J. Protein microarrays for multiplex analysis of signal transduction pathways. Nat. Med. 2004, 10, 1390-1396.

[27] Deng, Y.; Zhu, X. Y.; Kienlen, T.; Guo, A. Transport at the air/water interface is the reason for rings in protein microarrays. J. Am. Chem. Soc. 2006, 128, 2768-2769.

[28] Jiang, X. Y.; Xu, Q.; Dertinger, S. K. W.; Stroock, A. D.; Fu, T.; Whitesides, G. M. A general method for patterning gradients of biomolecules on surfaces using microfluidic networks. Anal. Chem. 2005, 77, 23382347.

[29] McDonald, J. C.; Duffy, D. C.; Anderson, J. R.; Chiu, D. T.; Wu, H.; Schueller, O. J.; Whitesides, G. M. Fabrication of microfluidic systems in poly(dimethylsiloxane). Electrophoresis 2000, 21, 27-40.

[30] Whitesides, G. M. The origins and the future of microfluidics. Nature 2006, 442, 368-373.

[31] Psaltis, D.; Quake, S. R.; Yang, C. Developing optofluidic technology through the fusion of microfluidics and optics. Nature 2006, 442, 381-386.

[32] Bernard, A.; Michel, B.; Delamarche, E. Micromosaic immunoassays. Anal. Chem. 2001, 73, 8-12.

[33] Wolf, M.; Juncker, D.; Michel, B.; Hunziker, P.; Delamarche, E. Simultaneous detection of C-reactive protein and other cardiac markers in human plasma using micromosaic immunoassays and self-regulating microfluidic networks. Biosens. Bioelectron. 2004, 19, 1193-1202.

[34] Gyorvary, E. S.; O’Riordan, A.; Quinn, A. J.; Redmond, G.; Pum, D.; Sleyt, U. B. Biomimetic nanostructure fabrication: Nonlithographic lateral patterning and self-assembly of functional bacterial S-layers at silicon supports. Nano Lett. 2003, 3, 315-319.

[35] Goldman, E. R.; Balighian, E. D.; Mattoussi, H.; Kuno, M. K.; Mauro, J. M.; Tran, P. T.; Anderson, G. P.; Avidin: A natural bridge for quantum dot-antibody conjugates. J. Am. Chem. Soc. 2002, 124, 6378-6382.

[36] Jaiswal, J. K.; Goldman, E. R.; Simon, S. M. Use of quantum dots for live cell imaging. Nat. Methods 2004, 1 , 73-78. 